\title{
Alprazolam withdrawal delirium
}

\author{
Karunaratne R, Prasangika WADR
}

District General Hospital, Matara

Alprazolam is a benzodiazepine often prescribed to treat insomnia and anxiety symptoms. ${ }^{1}$ It is licensed to use in panic disorder and generalized anxiety disorder by FDA and is in the list of medications with price control in Sri Lanka.

Benzodiazepine receptors are coupled with GABA-A receptors, and by this mechanism benzodiazepines enhance the effects of GABA by increasing GABA affinity at the GABA receptor. ${ }^{2}$ The neurotransmitter GABA, when bound to the GABA receptor, mediates the calming or inhibitory effects of alprazolam on the nervous system. ${ }^{3}$

If a person has been on a benzodiazepine for more than three or four weeks, he or she is likely to experience withdrawal symptoms if the drug is stopped abruptly. ${ }^{1}$ Patients tend to develop dependence and tolerance to benzodiazepines on prolonged use. ${ }^{4}$ Long term use of benzodiazepines gives rise to dose escalation and worsening of the underlying condition. ${ }^{1}$

Unlike other benzodiazepines alprazolam has more abuse potential due to its short half-life, rapid absorption, and low lipophilicity. Compared to other benzodiazepines, alprazolam effects may be felt within 30 minutes and can last for about 6 hours. Alprazolam taken in large doses are known to cause strong depressive effects which can result in memory loss.

We present a 65 year old retired bank officer with depressive disorder, who was admitted to the Psychiatry unit, District General Hospital, Matara in a state of withdrawal delirium after being on alprazolam for eight years.

\section{Case history -}

Mr. D. G. Sarath is a 65 year old retired Bank Officer, married with two children, from Dickwella, near Matara. He had visited a general practitioner eight years ago with the complaint of waking up several times during sleep. He was prescribed alprazolam for two weeks. Mr. Sarath had started using alprazolam from time to time presenting that prescription to the pharmacy. The dose of alprazolam he needed to have a good sleep increased gradually.

Three months prior to hospital admission, family members had accompanied Sarath to a consultant psychiatrist with complaints of waking up three hours prior to the usual time, loss of appetite and suspiciousness about family members. He was diagnosed to have a severe depressive episode with psychotic symptoms and started on mirtazapine and risperidone combination. The Psychiatrist got to know that Mr. Sarath has been taking alprazolam to improve sleep for years. After explaining Sarath and family members the harmful effects of long term use of alprazolam and the drug was replaced with diazepam which is a long acting benzodiazepine, with the aim of gradually withdrawing him from regular benzodiazepine use.

However Sarath started refusing newly started medications including diazepam and his worsening symptoms prompted his wife to admit him to the General Hospital, Matara. He had continued to take alprazolam to get rid of sleep disturbances. In the hospital alprozolam was stopped and he 
developed clear features of benzodiazepine withdrawal. Last day of his alprazolam use was a day prior to the hospitalization. On questioning it was clear that Mr. Sarath was dependent on alprazolam; the dose of alprazolam he needed to have a good sleep had increased gradually, there was a compulsion to take alprazolam and he revealed that he had been experiencing anxiety symptoms if he failed to take alprazolam even for one or two days.

Features of Benzodiazepine withdrawal including anxiety symptoms such as excessive sweating, tremors and irritability, perceptual disturbances like hypersensitivity to stimuli and derealization and other features namely persecutory delusions and suicidal ideas were present. He was having marked tremors, visual hallucinations involving faces and clouding of consciousness. Pulse rate was 120 beats per minute; body temperature was $99^{\circ} \mathrm{F}$. Mr. Sarath was disoriented in time, place and person. His attention and concentration was poor; short-term memory and long-term memory were impaired. These features were suggestive of the diagnosis of delirium tremens.

According to the benzodiazepine withdrawal scale Mr. Sarath was in a severe withdrawal with a total score of 57. He was irritable, anxious, weak and agitated. He complained of loss of appetite, poor sleep, fearfulness, fatigue, muscle aches and palpitations was feeling tensed, upset and worried of possible misfortunes in the future. Concentration was poor; he was restless at night.

Examination of the respiratory system, abdomen and genito-urinary system did not reveal any abnormality. Complete blood count, serum electrolytes, blood urea and creatinine, urine full report, random plasma glucose and liver function tests were found to be normal. Workup for sudden onset of altered mental status revealed no abnormal results.

He had not been diagnosed to be mentally ill prior to diagnosis of depressive disorder with psychotic features 3 months before hospitalization. There was no history of self-harm. He has had no contact with law enforcing authority. Mr. Sarath used to consume one glass of arrack at social gatherings; and his last alcohol intake was three months ago. He has been consuming three or four cigars per day for the past ten years. He was living with wife and two children at their own place. There were no financial hardships or family conflicts.

During hospital stay alprozolam was stopped and replaced with chlordiazepoxide because the latter is a benzodiazepine with a long half life and less severe withdrawal symptoms. Chlordiazepoxide 20 $\mathrm{mg}$ mane, $20 \mathrm{mg}$ vesper and $40 \mathrm{mg}$ nocte was started on the first day; it was tailed off gradually over seven days. Pulse rate, blood pressure and body temperature were monitored. He was examined daily to assess tremors, hallucinations and cognitive functions. Withdrawal symptoms responded to detoxification with chlordiazepoxide. The patient became afebrile with a normal pulse rate and normal cognition. He was free of visual hallucinations by 1 week.

Severe depressive episode with psychotic symptoms was treated with risperidone $2 \mathrm{mg}$ nocte and mirtazapine $15 \mathrm{mg}$ nocte which was continued after hospital discharge. He was educated on maintaining good sleep hygiene by avoiding caffeine, nicotine and alcohol after dinner, avoiding heavy meals at night and minimizing light and noise in bedroom. Clinic follow up was arranged at the District General Hospital, Matara for the management of Depressive disorder.

\section{Discussion -}

Long term use of benzodiazepines is associated with harmful effects like dependency and cognitive decline. Over the last 20 years, the quantity of benzodiazepines on each prescription has increased. ${ }^{1}$ Alprazolam is the second most popular drug increasing more than eight fold. The risk of inducing dependence can be reduced by issuing prescriptions limited to one or two weeks supply. ${ }^{1}$ Switching to a long acting benzodiazepine is helpful in the management of benzodiazepine withdrawal and 
maintenance treatment. Withdrawal tends to be severe if the patient has been on a benzodiazepine with a short half life. Abrupt cessation of benzodiazepines after a period of 1-6 months of use can cause life threatening seizures, so dose has to be reduced gradually. ${ }^{1}$ Most studies in primary care have found that gradual withdrawal over at least ten weeks is successful in achieving long term abstinence.

\section{References:}

1. Brett J, Murnion B. Management of Benzodiazepine misuse and dependence. Australian Prescriber. 2015 Oct;38:152-155

2. Masiulis $S$, Desai $R$, Uchański $T$, et al. $G A B A_{A}$ receptor signalling mechanisms revealed by structural pharmacology. Nature. 2019 Jan;565(7740):454-459

3. McKenzie RR, Jr, Madadi P, Sharma TR. Alprazolam withdrawal delirium and brief reversible psychosis: a case report. Prim Care Companion CNS Disord. 2014:16 